Missed Diagnosis

\title{
Lipomatosis of the ileocaecal valve simulating Crohn's disease
}

\author{
A.J. Bhupalan, A. Forbes, E. Lloyd-Davies, B. Wignall ${ }^{1}$ and \\ I.M. Murray-Lyon
}

Gastrointestinal Unit and 'Department of Radiology, Charing Cross Hospital, Fulham Palace Road, London W6 $8 R F, U K$

\section{Summary: A case of lipomatosis of the ileocaecal valve mimicking Crohn's disease of the terminal ileum is reported.}

\section{Introduction}

The radiological appearances of the terminal ileum seen in Crohn's disease are usually characteristic but some other conditions may mimic these features. These include infections like tuberculosis, ${ }^{1}$ Yersinia enterocolitica ${ }^{2}$ and more rarely actinomycosis as well as neoplastic causes like adenocarcinoma, ${ }^{3}$ lymphosarcoma ${ }^{4}$ and carcinoid. ${ }^{5}$ We describe a case of lipomatosis of the ileocaecal valve simulating Crohn's disease.

\section{Case report}

A 21 year old non-obese female initially presented with a ten month history of intermittent colicky right iliac fossa pain associated with diarrhoea. There were no specific aggravating or relieving factors.

General examination was unremarkable and sigmoidoscopy to $12 \mathrm{~cm}$ was normal.

Routine blood tests revealed a normal full blood profile, ESR, liver function tests, serum urea and electrolytes. Stool microscopy and culture were negative for parasites and pathogens.

A barium follow through revealed a constant irregular appearance in the distal $7 \mathrm{~cm}$ of the terminal ileum (Figure 1). The wall adjacent to the caecum appeared flat with a polypoid appearance to the medial wall. Overall the appearance was consistent with Crohn's disease. Colonoscopy showed a normal large bowel up to the ileocaecal valve which was narrow and distorted. No views of

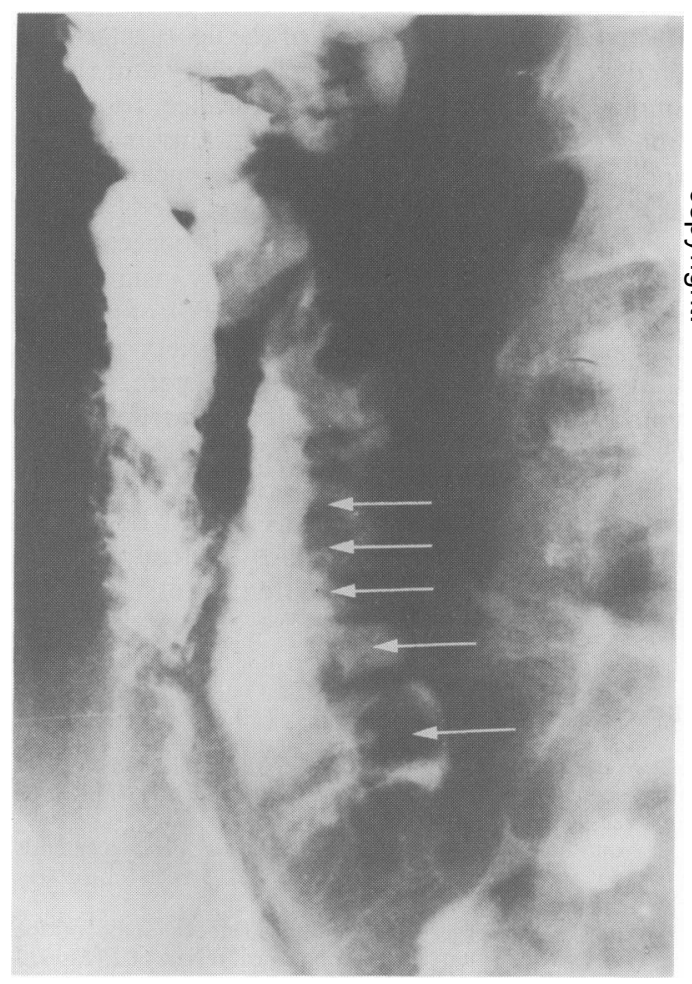

Figure 1 Terminal $7 \mathrm{~cm}$ of ileum shows polypoidal thickening with widening of the mucosal folds (see arrows). Normal caecum in spasm.

the terminal ileum were obtained. Biopsies taken from around the ileocaecal valve revealed nonspecific inflammatory changes histologically. 
She was treated with corticosteroids with little symptomatic benefit and after 18 months was referred for surgery. At laparotomy there were no external signs of Crohn's disease; however, there was thought to be some narrowing at the ileocaecal valve. A limited right hemicolectomy was performed with a side to side anastomosis. Macroscopic and histological examination of the specimen revealed appearances consistent with lipomatosis of the ileocaecal valve (Figure 2) as well as ulceration and hyperplastic changes in the ileum, probably secondary to intermittent intussusception. Since the operation she has remained asymptomatic.

\section{Discussion}

Lipomatosis of the ileocaecal valve, also known as lipohyperplasia, is characterized by diffuse annular infiltration of fatty tissue, mainly in the submucous layer. Its lack of encapsulation differentiates it from a lipoma. Lipomatosis of the ileocaecal valve tends to be more common in females, ${ }^{6}$ and this is similar to the female preponderance found in lipomas of the gastrointestinal tract. Lipomatosis is rare under the age of $40^{6}$ and appears to be associated with generalized obesity.

Many cases of lipomatosis of the ileocaecal valve are asymptomatic or give rise to only insignificant symptoms. Kelby ${ }^{7}$ found that only $50 \%$ of patients had any clinical manifestations. A variety of symptoms may occur including abdominal pain, nausea, vomiting, abdominal distension, diarrhoea and constipation. Rarely, gastrointestinal bleeding is seen and melaena has been described. ${ }^{8}$ The term ileocaecal valve syndrome was coined by Lasser and Rigler ${ }^{9}$ to describe a clinical picture which is presumably based on intermittent intussusception

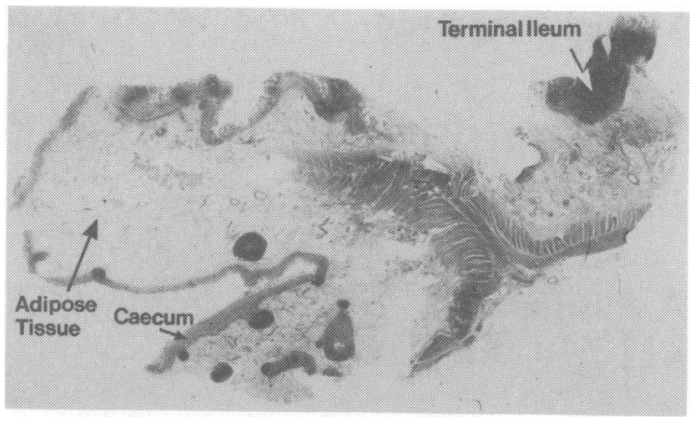

Figure 2 This microscopic slide reveals increased adipose tissue in the region of the ileocaecal valve as shown by the arrow. Magnification $\times 2$.

and chronic partial obstruction.

The most common causes of a prominent ileocaecal valve are lipomatosis, ${ }^{6,9}$ prolapse of the ileocaecal valve into the caecum, ${ }^{10}$ lymphoma, ${ }^{9}$ and oedema which may be associated with inflammatory bowel disease, especially Crohn's disease. There has also been a case report of Crohn's ileo-colitis associated with lipomatosis of the ileocaecal valve. ${ }^{11}$

Diagnosis may be difficult, as in our case. On barium enema, lipomatosis of the ileocaecal valve may appear as a translucent shadow. ${ }^{5}$ It is sometimes difficult to distinguish this from a polyp, a benign tumour and even a carcinoma. Colonoscopy can be helpful, as the ileocaecal valve may be visualized and biopsied.

Surgical resection with ileo-colic anastomosis is the only effective treatment and should be considered for symptomatic relief or when the diagnosis is uncertain. If the patient is asymptomatic, no treatment is required.

\section{References}

1. Werbeloff, L., Novis, B., Bank, S. et al. The radiology of tuberculosis of the gastrointestinal tract. Br J Radiol 1973, 46: 329-336.

2. Vantrappen, G., Agg, H., Ponette, E. et al. Yersinia enteritis and enterocolitis: gastroenterological aspects. Gastroenterology 1977, 72: 220-227.

3. Loie, T. \& Biggart, J. Primary adenocarcinoma of the jejunum and ileum: clinicopathological review of 25 cases. $J$ Clin Pathol 1990, 43: 533-536.

4. Puri, P. \& Guiney, E. Small bowel tumours causing intussusception in childhood. Br J Surg 1985, 72: 493-494.

5. Schnur, M. \& Seaman, W. Prolapsing tumours of the terminal ileum simulating enlarged ileocaecal valves. $\mathrm{Am} \mathrm{J}$ Roentgenol 1980, 134: 1133-1136.

6. Boquist, L., Bergdahl, L. \& Anderson, A. Lipomatosis of the ileocaecal valve. Cancer 1972, 29: 136-140.

7. Kelby, G.M. Submucous lipomas of the ileocaecal valve. Lancet 1948, 68: 301-304.

8. Hovelius, L. Lipomatosis of the ileocaecal valve. Acta Scand Chir 1973, 139: 673-676.

9. Lasser, E.C. \& Rigler, L.C. Ileocaecal valve syndrome. Gastroenterology 1955, 28: 1-161.

10. Vanlierde, M. \& Kahn, D. Hypertrophic ileocaecal valve stimulating a malignant tumour. $S$ Afr Med $J$ 1987, 72: $50-51$.

11. Berk, R.N., Davis, G.B. \& Cholassey, E.B. Lipomatosis of the ileocecal valve. Am J Roentgenol 1973, 119: 323-328. 